\title{
Evaluación de la coloración de Albert para la identificación de Corynebacterium spp. en flujo vaginal
}

\author{
Evaluation of Albert's staining technique for \\ Corynebacterium spp. in vaginal swabs
}

\begin{abstract}
Adriana M. Botero-Vélez Bact ${ }^{1}$, Ana C. Ossa-Giraldo PhD², Mary L. Vélez-Restrepo MSc ${ }^{3}$, Claudia M. Cuervo-Araque MSc4, Xiomara Úsuga-Perilla $M S^{5}$
\end{abstract}

Introducción: la detección del género Corynebacterium en flujo vaginal representa un reto para el estudio de su posible papel patogénico en el tracto genital femenino. La coloración de Gram es la técnica recomendada para la detección de corinebacterias en muestras clínicas; no obstante, algunas características biológicas de estas bacterias dificultan la identificación por medio de esta. Objetivo: evaluar el desempeño de la coloración de Albert para la identificación de Corynebacterium spp. en muestras de flujo vaginal. Materiales y métodos: se evaluó la tinción de Albert como prueba diagnóstica para la identificación de Corynebacterium spp. en 451 flujos vaginales en comparación de la coloración de Gram. Se calculó la sensibilidad, la especificidad, los valores predictivos, las razones de verosimilitud y el indice J de Youden con el software Epidat 3.1. Resultados: la coloración de Albert presentó sensibilidad y especificidad de $61,5 \%$ y $74,6 \%$, respectivamente, valor predictivo positivo de $78,3 \%$, valor predictivo negativo de 56,5\%, razón de verosimilitud positiva de 2,4 y negativa de 0,5 y un Índice de Youden de 0,4. Conclusiones: la tinción de Albert no mostró un mejor rendimiento que la coloración de Gram en la identificación del género Corynebacterium en muestras de flujo vaginal. Por lo tanto, se recomienda el uso en conjunto de las coloraciones de Gram y Albert para la identificación de corinebacterias en flujo vaginal.

Palabras claves: Corynebacterium, difteroides, coloración y etiquetado, tinción para gránulos metacromáticos, coloración de Gram, excreción vaginal.

${ }^{1}$ Bacterióloga y laboratorista clínica, especialista en Microbiología Clínica. Bacterióloga, IPS Incorpore, Institución Universitaria Colegio Mayor de Antioquia.

${ }^{2}$ Microbióloga y Bioanalista, especialista en Microbiología Clínica, candidata a PhD en Ciencias Básicas Biomédicas. Profesora Facultad de Medicina, Universidad Cooperativa de Colombia-sede Medellín, y Facultad Ciencias de la Salud, Institución Universitaria Colegio Mayor de Antioquia. Grupo de Investigación Infettare, Facultad de Medicina, Universidad Cooperativa de Colombia-sede Medellín y Grupo de Investigación Biociencias, Facultad Ciencias de la Salud, Institución Universitaria Colegio Mayor de Antioquia. Medellin, Colombia. Correspondencia: Calle 50 N. ${ }^{\circ}$ 40-74, $4^{\circ}$ piso. Teléfono: 57

44446065 ext.4965 - 4236. Correo electrónico: ana.ossag@campusucc.edu.co, ana.ossa@colmayor.edu.co

${ }^{3}$ Bacterióloga y Laboratorista Clínico, especialista y MSc en Microbiología Clínica. Profesora Facultad Ciencias de la Salud, Institución Universitaria Colegio Mayor de Antioquia. Medellin, Colombia.

${ }^{4}$ Bacterióloga y Laboratorista Clínico, especialista y MSc en Epidemiología. Profesora Facultad Ciencias de la Salud, Institución Universitaria Colegio Mayor de Antioquia. Grupo de Investigación Biociencias, Facultad Ciencias de la

Salud, Institución Universitaria Colegio Mayor de Antioquia. Medellin, Colombia.

${ }^{5}$ Bacterióloga y Laboratorista Clínico, MSc en Biología. Profesora Facultad de Medicina, Universidad Cooperativa de Colombia sede Medellin. Grupo de Investigación Infettare, Facultad de Medicina, Universidad Cooperativa de Colombia sede Medellín. Medellín, Colombia.

Conflicto de intereses: los autores declaran que no tienen conflicto de intereses Medicina \& Laboratorio 2017; 23: 179-186

Módulo 19 (Investigación), número 56. Editora Médica Colombiana S.A. $2017^{\odot}$

Recibido el 04 de abril de 2017; aceptado el 23 de abril de 2017 
Botero-Vélez AM, Ossa-Giraldo AC, Vélez-Restrepo $M L$, Cuervo-Araque CM, Úsuga-Perilla X. Evaluación de la coloración de Albert para la identificación de Corynebacterium spp. en flujo vaginal. Medicina \& Laboratorio 2017; 23: 179-186

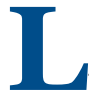

as especies de Corynebacterium diferentes a Corynebacterium diphtheriae, conocidas como difteroides o corinebacterias, son reconocidas por ser bacilos Gram positivos, pleomórficos, inmóviles, no endosporados, productores de catalasa y formadores de gránulos metacromáticos; algunos aerobios exclusivos y en su mayoría anaerobios facultativos [1,2]. Este género bacteriano ha sido considerado desde mucho tiempo atrás parte de la microbiota humana saprófita de la piel y las mucosas; no obstante, existen evidencias de su papel patogénico en pacientes inmunosuprimidos $[3,4]$, con dispositivos protésicos [5] y con estancias prolongadas en hospitales, en los que produce infecciones como linfadenitis granulomatosa, neumonitis, faringitis, infecciones de la piel, endocarditis y prostatitis, entre otros [6-9]. Además, se ha sugerido un rol patogénico de diferentes especies de corinebacterias en el sistema genitourinario de individuos inmunocompetentes; incluso, en algunas situaciones han sido asociadas a infecciones tanto en hombres como mujeres, como es el caso de las especies Corynebacterium riegelii y Corynebacterium urealyticum, y han sido implicadas en complicaciones del embarazo (Corynebacterium aurimucosum) [9], aborto espontáneo (Corynebacterium nigricans) [10] y vaginitis en una paciente preadolescente (Corynebacterium amycolatum) [11].

Por otro lado, hay especies que han sido aisladas del sistema genitourinario o sus fluidos corporales sin certeza sobre su patogenicidad como Corynebacterium freneyi de semen y tracto genital femenino, Corynebac- terium glucuronolyticum de semen y tracto genital masculino, Corynebacterium lipophiloflavum de secreción vaginal, Corynebacterium singulare de semen, Corynebacterium tuberculostericum de uretra, orina y tracto urogenital, Corynebacterium macginleyi y Corynebacterium pseudodiphtheriticum de orina[9] y Corynebacterium minutissimum y Corynebacterium amycolatum del tracto genitourinario [12]. En nuestro medio también se han podido evidenciar corinebacterias en flujo vaginal tanto de pacientes con sintomatología clínica [13] como en mujeres de la población general [14].

En la actualidad, los laboratorios de microbiología especializados utilizan varias técnicas para la identificación de Corynebacterium spp., algunas de estas basadas en la identificación fenotípica, como el API Cory 2.0, el API $50 \mathrm{CH}$ plus, el API 20E (BioMérieux, MarcyI'Étoile, Francia) y el RapID ${ }^{\text {TM }}$ CB PLUS System (Thermo Scientific, Massachusetts, Estados Unidos), en la secuenciación del gen rpoB o del gen del ARN ribosomal 16s, o en el perfil proteico de cada especie a través de la técnica de desorción/ionización mediante láser asistida por matriz (MALDI-TOF; del inglés, Matrix-Assisted Laser Desorption/IonizationTime-Of-Flight), entre otros [15-19].

A pesar de que estas pruebas tienen una alta sensibilidad y especificidad requieren de equipos especializados con altos costos para los laboratorios de primer nivel, lo que hace que su uso esté limitado a los laboratorios de referencia o investigación y, prácticamente, solo para la identificación de Corynebacterium diphtheriae, la cual, de manera clásica, ha sido considerada la especie de este género de mayor importancia clínica. De otro lado, la tinción de Gram ha sido recomendada para la detección de corinebacterias en fluidos corporales y tejidos [20]; no obstante, la identificación con esta 
técnica puede ser dificultosa debido a su pleomorfismo y no se encuentran estudios en la literatura científica que hayan evaluado su rendimiento para el diagnóstico de estas bacterias.

Teniendo en cuenta lo anterior, sumado a la evidencia de la circulación de Corynebacterium spp. en nuestra región y el papel patogénico emergente de los difteroides, se hace necesario buscar y evaluar técnicas que permitan hacer una detección acertada, rápida y económica de este género en el tracto genital femenino, las cuales, en conjunto con la tinción de Gram para la identificación de los otros géneros presentes en ese sitio anatómico, permitan tener un panorama preciso de la microbiota vaginal de las pacientes y optimizar su diagnóstico. Una opción para esto es la tinción de Albert, la cual ha sido utilizada durante mucho tiempo para el diagnóstico e identificación de Corynebacterium diphtheriae, pero hay evidencia de que podría ser empleada para la identificación de otras corinebacterias [21]. Con base en lo anterior, el objetivo de este estudio fue evaluar si la coloración de Albert podría tener un mejor rendimiento que la tinción de Gram en la identificación de Corynebacterium spp. en muestras de flujo vaginal.

\section{Materiales y métodos}

\section{Tipo y población de estudio}

Se realizó un estudio descriptivo para la evaluación de una prueba diagnóstica, usando como fuente de información primaria muestras de flujo vaginal y datos demográficos y clínicos de 451 mujeres residentes del Valle de Aburrá, Antioquia, seleccionadas por conveniencia de ocho instituciones de educación superior del municipio de Medellín, una fundación de mujeres y un grupo de mujeres de un barrio de la ciudad de Medellín, durante los años 2012 y 2013. Se reclutaron mujeres que, previa información y firma del consentimiento informado, aceptaron de forma voluntaria participar en la investigación, a las cuales se les aplicó una encuesta para determinar sus características sociodemográficas. Como criterio de inclusión se estableció que fueran mujeres mayores de edad y de exclusión la presencia del sangrado menstrual al momento de la toma de la muestra y que nunca hayan tenido relaciones sexuales. A las participantes se les brindaron los resultados del examen de flujo vaginal (directo y tinción de Gram) y de la citología cérvico-vaginal, realizadas por laboratorios habilitados de la ciudad, como reciprocidad y beneficio de su participación en el estudio.

\section{Detección de Corynebacterium spp. en flujo vaginal}

Por cada participante se hicieron dos extendidos a partir del mismo exudado vaginal, que fueron sometidos a la tinción de Gram y a la coloración de Albert para determinar la presencia de Corynebacterium spp. y evaluar el desempeño de la técnica de Albert en comparación con la tinción de Gram, que es la prueba de referencia sugerida por la Asociación Americana de Microbiología (ASM; del inglés, American Society for Microbiolo$g y)$ [6]. Las muestras fueron teñidas según el protocolo definido por el fabricante para las coloraciones de Gram y Albert (IHR Diagnostica Ltda., Cali, Colombia) $[22,23]$ y su lectura se realizó con el objetivo de 100X, reportando ausencia o presencia de corinebacterias.

Para controlar los posibles sesgos en la recolección de la información, el procesamiento y la lectura fueron ciegos y realizados de 
manera independiente por dos observadores distintos. Para garantizar la confiabilidad del diagnóstico se estandarizaron ambas tinciones con la cepa Corynebacterium urealyticum, identificada con el código 43044 y obtenida del Centro de Biorecursos ATCC (del inglés, American Type Culture Cell). Esta misma cepa se usó como control en los ensayos.

\section{Análisis estadístico}

Para la coloración de Albert se estimó su desempeño y eficiencia a partir de los valores predictivos positivo y negativo (VPP y VPN) y la razón de verosimilitud positiva y negativa (RVP y RVN). La eficiencia de la prueba se analizó con el índice J de Youden y la concordancia entre los observadores con el índice de Kappa (escala de interpretación: 0,00=pobre, 0,01-0,20=leve, 0,214-0,40=aceptable, $0,41-0,60=$ moderada, $0,61-0,80=$ conside rable y $0,81-1,00=$ casi perfecta [24]). Todos estos parámetros, incluyendo los respectivos intervalos de confianza del 95\%, fueron calculados con el programa EPIDAT versión 3.1 (Dirección Xeral de Saúde Pública, Xunta de Galicia, España) y su interpretación estuvo basada en trabajos previos sobre pruebas diagnósticas [25-27].

\section{Aspectos éticos}

La recolección de las muestras se hizo previo aval del Comité de Bioética de la Universidad Cooperativa de Colombia, sede Medellín (Colombia) y se siguieron los lineamientos de la resolución 8430 de 1993 del Ministerio de Salud de la República de Colombia y la Declaración de Helsinki.

\section{Resultados}

Las mujeres incluidas en el estudio tenían edades entre los 18 y 76 años, con un pro- medio de edad de 26 años (desviación estándar=10, mediana $=22$, rango intercuartílico=19-29). La mayoría de las mujeres pertenecían a los estratos socioeconómicos 2, 3 y 4. La estratificación socioeconómica de la población en Colombia se hace sobre los inmuebles residenciales y comprende desde el estrato 1 al 6 , siendo 1 el estrato económico más bajo y 6 el estrato económico más alto [28].

La concordancia en la lectura de los extendidos con la coloración de Gram entre los dos observadores fue considerable (índice Kappa=0,723), y para la tinción de Albert fue aceptable (índice Kappa=0,33). La prevalencia de Corynebacterium spp. fue de $59,9 \%$ en las muestras analizadas con la prueba de referencia, la tinción de Gram, mientras que con la coloración de Albert fue de $47,0 \%$. En la tabla 1 se presentan los resultados de la identificación de la bacteria con la coloración de Albert respecto a la tinción de Gram.

De acuerdo con estos resultados, la coloración de Albert tuvo una sensibilidad de $61,5 \%$, especificidad de $74,6 \%$ y valores predictivos positivo y negativo de $78,3 \%$ y $56,5 \%$, respectivamente, y una eficiencia moderada $(66,7 \%)$. Asimismo, de acuerdo con el índice de Youden hallado esta prueba no mostró una calidad o valor informativo aceptable (véase tabla 2).

\section{Discusión}

En el presente estudio se incluyeron 451 muestras de flujo vaginal de mujeres de la población general del Valle de Aburrá de Antioquia (Colombia), de las cuales el 59,9\% fueron positivas para la presencia de Corynebacterium spp. mediante la coloración de Gram y el $47,0 \%$ por la de Albert. La Asocia- 
Tabla 1. Identificación de Corynebacterium spp. con las tinciones de Gram y Albert $(n=451)$

\begin{tabular}{|c|c|c|c|}
\hline \multirow{2}{*}{ Coloración Albert } & \multicolumn{2}{|c|}{ Tinción Gram } & \multirow{2}{*}{ Total } \\
\hline & Positiva & Negativa & \\
\hline Positiva & 166 & 46 & 212 \\
\hline Negativa & 104 & 135 & 239 \\
\hline Total & 270 & 181 & 451 \\
\hline
\end{tabular}

\begin{tabular}{lll}
\hline $\begin{array}{l}\text { Tabla 2. Evaluación de la tinción de Albert } \\
\text { para el diagnóstico de Corynebacterium spp. }\end{array}$ \\
\hline Parámetro & Valor & $\begin{array}{l}\text { Intervalo de } \\
\text { confianza } \\
\text { del 95\% }\end{array}$ \\
\hline Sensibilidad (\%) & 61,5 & $55,5-67,5$ \\
Especificidad (\%) & 74,6 & $68,0-81,2$ \\
Valor predictivo positivo (\%) & 78,3 & $72,5-84,1$ \\
$\begin{array}{l}\text { Valor predictivo negativo (\%) } \\
\text { Razón de verosimilitud }\end{array}$ & 56,5 & $50,0-63,0$ \\
positiva & 2,4 & $1,9-3,2$ \\
$\begin{array}{l}\text { Razón de verosimilitud } \\
\text { negativa }\end{array}$ & 0,5 & $0,4-0,6$ \\
Eficiencia & 66,7 & $62,3-70,9$ \\
Índice de Youden & 0,4 & \\
Índice Kappa & 0,33 & \\
\hline
\end{tabular}

ción Americana de Microbiología recomienda para la identificación de Corynebacterium spp. la coloración de Gram [20]; sin embargo, hasta nuestro conocimiento no se cuenta a la fecha con estudios que hayan evaluado su rendimiento para el diagnóstico de estas bacterias.

Es reconocido que la identificación de las corinebacterias utilizando la tinción de Gram se ve afectada por el pleomorfismo entre las diferentes especies [20], la irregularidad en la captación de la tinción, que puede estar relacionada con la composición bioquímica de la bacteria (p. ej. la concentración de ácidos micólicos) [29-31], la presencia de poros [32], su estadio de vida [33], la temperatura de crecimiento y la disposición de nutrientes en el medio en el que se encuentre [34]; lo que lleva a que no haya un solo patrón morfológico y de reacción tintorial al Gram, sino una variedad de patrones que generan confusión durante la identificación de estos microorganismos por el personal de laboratorio, aun en aquellos de larga trayectoria en el área.

Debido a estas dificultades que se presentan en el uso de la tinción de Gram y a la evidencia del potencial patogénico en el tracto genitourinario femenino de las especies de Corynebacterium [10,12], en este estudio se evaluó la validez de coloración de Albert para la identificación de estas bacterias como una posible opción para la optimización de su diagnóstico. La sensibilidad de la coloración de Albert fue baja (61,5\%), lo que indica que no detecta correctamente todos los casos positivos para Corynebacterium spp. determinados al Gram, aunque mostró un valor de especificidad mejor $(74,6 \%)$, indicativo de que detecta correctamente las muestras negativas para Corynebacterium spp. Por su parte, se encontró, en una buena proporción, que un resultado positivo por esta prueba confirma que en efecto hay infección por la bacteria (valor predictivo positivo $=78,3 \%$ ), pero que un resultado negativo no descarta que la mujer esté realmente infectada (valor predictivo negativo $=56,5 \%$ ), por lo que su validez fue deficiente.

Aunque los resultados de este estudio arrojaron un $25,4 \%$ de falsos positivos en la coloración de Albert, es posible que estos no correspondan exclusivamente a fallas del método, sino que por el contrario sean corinebacterias que fueron detectadas por la coloración de Albert y no por el Gram, dado que esta última tinción no permite la identificación de los gránulos metacromáticos que colorea específicamente el Albert [21] y que son característicos de este género bacteriano [9]. Estos gránulos, generalmente 
de reserva, están constituidos, en el caso de Corynebacterium diphteriae y otras corinebacterias, por componentes ácidos como los polifosfatos (volutina) y por otros compuestos como el ácido beta amino butírico o los polímeros de glutamato en otras especies. Con la coloración de Albert los gránulos metacromáticos se tiñen de color rojo, violeta o diferentes tonos de azul al entrar en contacto con el azul de toluidina, razón por la cual se les llaman metacromáticos [21]. Paradójicamente, una explicación a la baja sensibilidad encontrada para el Albert en estas muestras se puede deber a la posibilidad de encontrar más de una especie de Corynebacterium spp. en la vagina y a que la formación y composición de los gránulos metacromáticos varía entre las especies y el estadio metabólico de las bacterias, lo que redunda en la probabilidad de que no se hayan detectado por el Albert aquellas corinebacterias que no expresaron gránulos metacromáticos, pero que sí fueron identificadas por el Gram.

El valor de la razón de verosimilitud positiva encontrada demostró que obtener un resultado positivo para corinebacterias por la coloración de Albert es dos veces más probable en una mujer infectada que en una mujer sana, mientras que la razón de verosimilitud negativa hallada mostró que la probabilidad de tener un resultado negativo por la coloración de Albert es solo 0,5 veces mayor en las mujeres sanas respecto a las infectadas, lo que significa que esta prueba-tiene baja capacidad para confirmar la presencia de infección por corinebacterias y, a su vez, de descartarla, por lo que es de poca ayuda para el clínico [25]. En cuanto a la eficiencia diagnóstica esta fue regular pues la tinción de Albert clasificó correctamente solo al $66,7 \%$ de los casos que fueron positivos por el Gram.
La coloración de Albert ha mostrado buen desempeño para la detección de Corynebacterium diphteriae [21,35], pero, a pesar de que se esperaba una tinción característica de las corinebacterias para su identificación [21], se observó, tanto en la estandarización como en el procesamiento de las muestras, que la cepa control (Corynebacterium urealyticum ATCC 43044) y los flujos vaginales analizados tomaron la coloración de manera tenue e irregular, lo que dificultó su identificación, a pesar de la capacitación previa de los observadores, lo que puede explicar la baja concordancia entre las lecturas del Albert. Además, el valor obtenido con el índice de Youden demuestra que la calidad del resultado con la tinción de Albert es baja.

La prevalencia de Corynebacterium spp. más alta se observó utilizando la coloración de Gram (60\%). Algunos estudios en Colombia han reportado prevalencias del $30 \%$ y $32 \%$ en mujeres consultantes por síntomas ginecológicos [13,35]; sin embargo, no es común encontrar investigaciones que reporten la prevalencia o frecuencia de este microrganismo en muestras de flujo vaginal en la población general, muy posiblemente debido a las fallas en su identificación mediante Gram, por las razones antes expuestas $y$, principalmente, a que la relevancia clínica de estas bacterias ha sido limitada, pues usualmente se consideran como parte de la microbiota de mucosas o de poca importancia clínica [36-38].

Los resultados de este estudio, sumados a la dificultad de usar otras pruebas de identificación más especializadas en los laboratorios de rutina, especialmente para muestras de flujo vaginal, permiten recomendar el uso en paralelo de las coloraciones de Gram y Albert para la detección de corinebacterias en este tipo de muestras, de forma que 
se disminuya la proporción de falsos negativos que se presentan al usar solo una de las dos coloraciones y, así mismo, aumentar la sensibilidad y los valores predictivos negativos del diagnóstico de estas infecciones.

Dado el emergente rol patogénico de Corynebacterium spp. en el tracto genitourinario, es necesario seguir en la búsqueda de otras opciones para su detección en flujo vaginal que ofrezcan las mismas ventajas económicas y de rapidez que las coloraciones de Gram y Albert, pero que superen sus debilidades inherentes a la biología de la bacteria, las cuales no son posibles de mitigar en su totalidad con entrenamiento del personal sanitario. Asimismo, se hace necesario realizar una correcta asociación de la presencia de Corynebacterium spp. con otros hallazgos microbiológicos, inmunológicos y clínicos, para evitar la subvaloración de la importancia patogénica de estas bacterias en algunos casos.

Una limitación de este estudio es que la muestra fue no probabilística, lo que impide que estos resultados puedan ser generalizados; no obstante, podrían trasladarse a otras poblaciones de características similares. Igualmente, no se estableció un límite inferior de detección de la coloración de Albert.

\section{Conclusiones}

De acuerdo con los resultados obtenidos, donde la coloración de Albert no tuvo un mejor rendimiento que el Gram, y a la dificultad de usar pruebas diagnósticas más especializadas en flujos vaginales $u$ otras pruebas no especializadas pero con mejor rendimiento, se recomienda el uso en conjunto de las coloraciones de Gram y Albert para la identificación de corinebacterias en flujo vaginal.

\section{Agradecimientos}

Este proyecto fue ejecutado con fondos del CONADI de la Universidad Cooperativa de Colombia y fondos de CICMA de la Institución Universitaria Colegio Mayor de Antioquia (Medellín, Colombia).

\section{Bibliografía}

1. Venezia J, Cassiday PK, Marini RP, Shen Z, Buckley EM, Peters $\mathrm{Y}$, et al. Characterization of Corynebacterium species in macaques. J Med Microbiol 2012; 61: 1401-1408.

2. Fernández Natal MI. Identificación y poder patógeno de microorganismos del género "Corynebacterium" aislados de muestras clínicas. Tesis para optar al título de Doctor en la Facultad de Medicina. Madrid, España: Universidad Complutense de Madrid; 2010.

3. Creagh R, Saavedra JM, Rodriguez FJ, Rodriguez P, Merino MD. [Pneumonia casued by Corynebacterium striatum in a patient with AIDS]. Enferm Infecc Microbiol Clin 2000; 18: 297298.

4. de Miguel I, Rodríguez E, Martín AM. Corynebacterium amycolatum: sepsis en pacientes hematológicos. Enferm Infecc Microbiol Clin 1999; 17: 340-341.

5. Cazanave C, Greenwood-Quaintance KE, Hanssen AD, Patel R. Corynebacterium prosthetic joint infection. J Clin Microbiol 2012; 50: 1518-1523.

6. Funke G, von Graevenitz A, Clarridge JE, 3rd, Bernard KA. Clinical microbiology of coryneform bacteria. Clin Microbiol Rev 1997; 10: 125-159.

7. Dalal A, Urban C, Segal-Maurer S. Endocarditis due to Corynebacterium amycolatum. J Med Microbiol 2008; 57: 1299-1302.

8. Bishai WR, Murphy JR. Diphtheria and Other Infections Caused by Corynebacteria and Related Species. En: Longo DL, Fauci AS, Kasper DL, Hauser SL, Jameson JL, Loscalzo J, eds. Harrison's Principles of Internal Medicine. Vol. 1 (ed 17a). Nueva York, Estados Unidos: McGraw-Hill; 2008: 890-895.

9. Bernard $\mathrm{K}$. The genus corynebacterium and other medically relevant coryneform-like bacteria. J Clin Microbiol 2012; 50: 3152-3158.

10. Shukla SK, Vevea DN, Frank DN, Pace NR, Reed KD. Isolation and characterization of a black-pigmented Corynebacterium $\mathrm{sp}$. from a woman with spontaneous abortion. J Clin Microbiol 2001; 39: 1109-1113.

11. Chen X, Zhao X, Chen L, Zeng W, Xu H. Vaginitis Caused by Corynebacterium amycolatum in a Prepubescent Girl. J Pediatr Adolesc Gynecol 2015; 28: e165-167.

12. Shukla SK, Bernard KA, Harney M, Frank DN, Reed KD. Corynebacterium nigricans sp. nov.: proposed name for a black-pigmented Corynebacterium species recovered from the human female urogenital tract. J Clin Microbiol 2003; 41: 4353-4358.

13. Castaño-Orozco ME, Ossa-Giraldo AC, Úsuga-Perilla X, Gil G. Prevalencia de Corynebacterium sp. en mujeres consultantes de una I.P.S de la ciudad de Medellín durante 2006-2012. CDROM de Memorias: $12^{\circ}$ Congreso Internacional del Colegio Nacional de Bacteriología CNB. Bogotá, Colombia: Colegio Nacional de Bacteriología CNB; 2012.

14. Botero AM, Úsuga $\mathbf{X}$, Cuervo $\mathbf{C M}$, Ossa $\mathbf{A C}$. Prevalencia de 
Corynebacterium spp. y factores asociados en mujeres del Valle de Aburrá. Acta Med Colomb 2015; 40: 234-240.

15. Engelkirk PG, Duben-Engelkirk JL. Laboratory Diagnosis of Infectious Diseases: Essentials of Diagnostic Microbiology. Filadelfia, Estados Unidos: Lippincott Williams \& Wilkins; 2008.

16. Hudspeth MK, Hunt Gerardo S, Citron DM, Goldstein EJ. Evaluation of the RapID CB Plus system for identification of Corynebacterium species and other gram-positive rods. J Clin Microbiol 1998; 36: 543-547.

17. Khamis A, Raoult D, La Scola B. rpoB gene sequencing for identification of Corynebacterium species. J Clin Microbiol 2004; 42: 3925-3931.

18. Drancourt M, Bollet C, Carlioz A, Martelin R, Gayral JP, Raoult D. $16 \mathrm{~S}$ ribosomal DNA sequence analysis of a large collection of environmental and clinical unidentifiable bacterial isolates. J Clin Microbiol 2000; 38: 3623-3630.

19. Alatoom AA, Cazanave CJ, Cunningham SA, Ihde SM, Patel R. Identification of non-diphtheriae corynebacterium by use of matrix-assisted laser desorption ionization-time of flight mass spectrometry. J Clin Microbiol 2012; 50: 160-163.

20. Versalovi J, Carroll KC, Funke G, Jorgensen JH, Landry ML, Warnock DW. Manual of Clinical Microbiology (ed 10a). Washington D.C., Estados Unidos: ASM Press; 2011.

21. Koneman EW, Allen SD, Janda WM, Schrenckenberger PC, Winn WC. Koneman. Diagnóstico microbiológico: Texto y Atlas en color. Bogotá D.C., Colombia: Editorial Médica Panamericana; 2001.

22. IHR Diagnóstica. Coloración GRAM. 2008. Disponible: http:// ihrdiagnostica.com/wp-content/uploads/2016/11/ColoracionGRAMv4-1.pdf Consultado: abr 2017.

23. IHR Diagnóstica. Coloración ALBERT. 2008. Disponible: http:// ihrdiagnostica.com/wp-content/uploads/2016/11/ColoracionALBERTv2.pdf. Consultado: abr 2017.

24. Landis JR, Koch GG. The measurement of observer agreement for categorical data. Biometrics 1977; 33: 159-174.

25. Burgos ME, Manterola C. Cómo interpretar un artículo sobre pruebas diagnósticas. Rev Chil Cir 2010; 62: 301-308.

26. Sousa MRd, Ribeiro ALP. Revisão sistemática e meta-análise de estudos de diagnóstico e prognóstico: um tutorial. Arq Bras Cardiol 2009; 92: 241-251.

27. Cuervo-Perez JF, Camilo Arango J, Cardona-Arias JA. Evaluación de técnicas inmunológicas in vitro para el diagnóstico de alergias: metanálisis 2000-2012. Rev Esp Salud Publica 2014; 88: 67-84.
28. República de Colombia, Departamento Administrativo $\mathrm{Na}$ cional de Estadística (Dane). Estratificación socioeconómica para servicios públicos domiciliarios. Disponible: http://www. dane.gov.co/index.php/servicios-al-ciudadano/servicios-deinformacion/estratificacion-socioeconomica. Consultado: abr 2017.

29. Bansal-Mutalik R, Nikaido H. Quantitative lipid composition of cell envelopes of Corynebacterium glutamicum elucidated through reverse micelle extraction. Proc Natl Acad Sci U S A 2011; 108: 15360-15365.

30. Meniche X, Labarre C, de Sousa-d'Auria C, Huc E, Laval F, Tropis $\mathbf{M}$, et al. Identification of a stress-induced factor of Corynebacterineae that is involved in the regulation of the outer membrane lipid composition. J Bacteriol 2009; 191: 73237332.

31. Gebhardt H, Meniche X, Tropis M, Kramer R, Daffe M, Morbach $\mathrm{S}$. The key role of the mycolic acid content in the functionality of the cell wall permeability barrier in Corynebacterineae. Microbiology 2007; 153: 1424-1434.

32. Soltan Mohammadi N, Mafakheri S, Abdali N, Barcena-Uribarri I, Tauch A, Benz R. Identification and characterization of the channel-forming protein in the cell wall of Corynebacterium amycolatum. Biochim Biophys Acta 2013; 1828: 2574-2582.

33. Kawata T, Inoue T. Electron Microscopic Observations of a Remarkable Body in Aged Corynebacterium Diphtheriae. J Bacteriol 1965; 89: 1613-1614.

34. Webster JA, Fay DD, Costa JL, Jones PM, Hugh R. Elemental composition of bacterial metachromatic inclusions determined by electron microprobe X-ray analysis. J Bacteriol 1984; 158: 441-446.

35. Palmerlee CA. Albert's Toluidin Blue as a Routine Stain for Diphtheria Bacilli. Am J Public Health (N Y) 1923; 13: 363-365.

36. Verstraelen H, Verhelst R, Roelens K, Claeys G, Weyers S, De Backer $\mathbf{E}$, et al. Modified classification of Gram-stained vaginal smears to predict spontaneous preterm birth: a prospective cohort study. Am J Obstet Gynecol 2007; 196: 528 e521-526.

37. Donders GG. Definition and classification of abnormal vaginal flora. Best Pract Res Clin Obstet Gynaecol 2007; 21: 355-373.

38. Bravo AB, Miranda LS, Lima OF, Cambas AV, Hernandez ML, Alvarez JM. Validation of an immunologic diagnostic kit for infectious vaginitis by Trichomonas vaginalis, Candida spp., and Gardnerella vaginalis. Diagn Microbiol Infect Dis 2009; 63: 257-260.

Introduction: The detection of Corynebacterium genus in vaginal discharge represents a challenge for the study of its possible pathogenic role in the female genital tract. Gram's stain is recommended for corynebacteria detection in clinical samples, however, some biological characteristics of these bacteria difficult their identification by this technique. Objetive: to evaluate Albert's stain performans to identify Corynebacterium spp. in vaginal discharge. Materials and methods: Albert's stain was evaluated as a diagnostic test for the identification of Corynebacterium spp. in 451 vaginal swabs in comparison to Gram's stain. Sensitivity, specificity, predictive values, likelihood ratio, as well as Youden's J index were calculated using Epidat software 3.1 version. Results: Albert's stain had a sensitivity and specificity of $61.5 \%$ and $74.6 \%$, respectively, positive predictive value of $78.3 \%$, negative predictive value of 56.5 $\%$, positive likelihood ratio of 2.4 and negative likelihood ratio of 0.5 , as well as Youden index of 0.4 . Conclusions: Albert's stain did not show better performance than Gram's stain for identification of corynebacteria in vaginal swabs. Thereforeit is recommended the concomitant use of the Gram and Albert stains for the identification of corynebacteria in vaginal discharge.

Keywords: Corynebacterium, diphtheroids, staining and labeling, metachromatic granules stain, Gram's stain, vaginal discharge. 